Measur ement of the gai $n$ vol ume in ampl ifying and scattering medi a by a two-beam spat i al - cor rel at i on net hod

\begin{tabular}{|c|c|}
\hline 著者 & I t o Tet su, Tomi t a Nakot o \\
\hline $\begin{array}{l}\text { j our nal or } \\
\text { publ i cat i on } \mathrm{titl} e\end{array}$ & Physi cal Revi ew E \\
\hline vol une & 63 \\
\hline number & 3 \\
\hline page $r$ ange & 036608-036608 \\
\hline year & 2001-02 \\
\hline 出版者 & Amer i can Physi cal Soci ety \\
\hline 権利 & (c) 2001 The Ameri can Physi cal Soci ety \\
\hline URL & ht t p: //hdl . handl e. net /10297/589 \\
\hline
\end{tabular}




\title{
Measurement of the gain volume in amplifying and scattering media by a two-beam spatial-correlation method
}

\author{
Tetsu Ito and Makoto Tomita \\ Department of Physics, Faculty of Science, Shizuoka University, 836 Ohya Shizuoaka, 422-8529 Japan
}

(Received 23 October 2000; published 21 February 2001)

\begin{abstract}
We measured the size of the gain volume at the threshold of the spectral collapse in a solution of kiton red with colloidal suspensions of polystyrene spheres using a two-beam spatial-correlation method. In this method, the luminescence spectrum was measured as a function of the spatial separation of two excitation beams. It was found that the size of the gain volume at the threshold $L_{t h}$, is proportional to the absorption length $L_{a}$, for different samples. Numerical simulations are also presented and the results are discussed on the basis of a relation $L_{t h}=\sqrt{3 G_{t h}} \sqrt{\sigma_{a} / \sigma_{s}} L_{a}$, where $\sigma_{s}$ and $\sigma_{a}$ are stimulated emission and absorption cross sections of kiton red, respectively.
\end{abstract}

DOI: 10.1103/PhysRevE.63.036608

PACS number(s): 42.25.Dd, 42.55.-f, 42.65.-k, 42.62.Fi

Light propagation in a multiple-scattering medium has been attracting increasing attention. When the medium has gain, luminescence in the medium experiences strong amplification by stimulated emission. This effect gives rise to the spectral collapse and random or diffusive laser action in the medium. Such an amplifying process was first discussed by Letokhov as an analogy of a nuclear reactor [1]. Lawandy et al. reported the observation of a spectral collapse and shortening of the lifetime in the luminescence from colloidal suspensions containing rhodamine 640 perchlorate dye in methanol and $\mathrm{TiO}_{2}$ particles, which resembled multimode laser behavior [2]. Random laser action depends on various factors [3-6]. When the mean-free-path becomes of the order of the wavelength, the luminescence light would coherently be feedbacked by the recurrent scattering processes $[7,8]$. Recently, Cao et al. reported discrete and angular dependent lasing modes in semiconductor powder and argued the possibility of coherent random laser [9].

It has been suggested that absorption saturation in the excitation process and the resultant increment in the gain volume is necessary to realize sufficient gain-path length for the random laser action [10]. The presence of the absorption saturation was experimentally well confirmed by the nonlinear reflectivity [11] and the excitation power dependent spectral redshifts and blueshifts in a strongly scattering medium [12]. The size of the gain volume could be examined from the excitation beam size dependence of the threshold energy of the spectral collapse $[13,14]$. It was found that there is a critical mean-free-path below which the threshold energy is almost independent of the mean-free-path for every excitation beam size. This behavior suggests that the diffusion volume of the luminescence light obtained with the critical mean-free-path is just the same order of the gain volume in the medium [13].

In this paper, we present experiments on the size of the gain volume in amplifying scattering media using a spatial correlation method, which gives direct information on the size of the gain volume. In this method, two excitation light pulses at the threshold energy of the spectral collapse are injected into the sample and the spectral width of the luminescence is measured as a function of the spatial separation between the first and second beams. When two beams are spatially overlapped within the gain volume, two beams work cooperatively to result in the strong spectral collapse. The spectral width as a function of the beam separation reflects the size of the gain volume in an amplifying and scattering medium. It was found that $L_{t h}$ is proportional to $L_{a}$ for different samples of the different transport mean-free-paths and the different absorption lengths. Numerical simulation based on the coupled rate and diffusion equations well recaptures the experimental results, which are discussed in terms of the stimulated emission cross section $\sigma_{s}$, and the absorption cross section $\sigma_{a}$, of dye molecules.

Figure 1 shows the schematic diagram of experimental setup, which is based on the Michelson interferometer. The excitation light source was the second harmonics of a injection seeded Q-switched $\mathrm{Nd}^{3+}$ yttrium-aluminum-garnet (YAG) laser. The wavelength was $532 \mathrm{~nm}$, the pulse width was $8 \mathrm{~ns}$, and the repetition rate was $5 \mathrm{~Hz}$. The excitation pulse energy was changed from $0.0007 \mathrm{~mJ}$ to $0.56 \mathrm{~mJ}$. The incident light beam was divided into two beams by a beam splitter (BS), and focused on the sample with the focusing lens L3. The beam diameter $w$, was $80 \mu \mathrm{m}$. The lens, $L 1$ and L2, were inserted in order to diverge the incident beam so that the two excitation beams make a separation distance $d$, at the focusing point of L3. One of the corner cube prisms $\mathrm{CP} 1$, was translated in order to change the beam separation $d$, at the focusing point of L3 on the surface of sample cell $S$. Two laser beams, B1 and B2, were orthogonally polarized by a

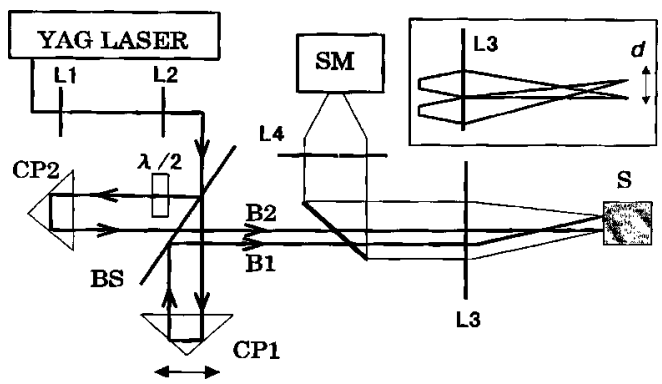

FIG. 1. Experimental setup. L1, L2, L3 and L4 are lenses; CP1 and $\mathrm{CP} 2$ are corner cube prisms; $\mathrm{BS}$ is the beam splitter; $\mathrm{SM}$ is the spectrometer; $\mathrm{S}$ is the sample cell. B1 and B2 are the first and second beams. 


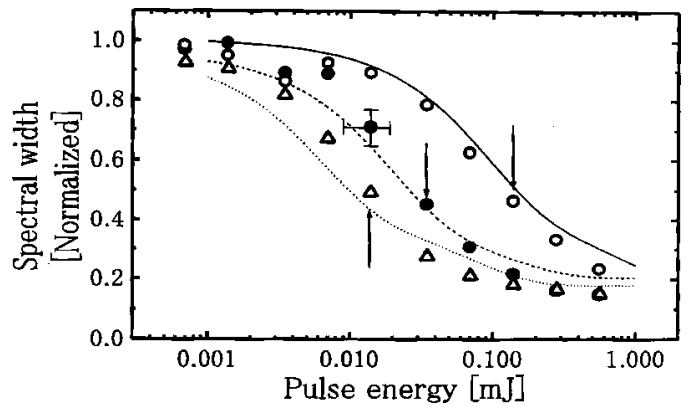

FIG. 2. Normalized spectral width of the luminescence light as a function of the excitation pulse energy. The concentrations of dye are $(\bigcirc) 2 \times 10^{-4} \mathrm{M},(\bigcirc) 5 \times 10^{-4} \mathrm{M}$, and $(\triangle) 10 \times 10^{-4} \mathrm{M}$ with the volume fractions of polystyrene spheres $2 \%, 5 \%$, and $10 \%$, respectively. The solid, dashed, and dotted lines are corresponding calculated curves.

$\lambda / 2$ wave plate in order to avoid the interference effect between two beams. The sample was methanol solution of kiton red with colloidal suspensions of polystyrene spheres. The concentration of dye was varied from $1 \times 10^{-4}$ to 10 $\times 10^{-4} \mathrm{M}$, which corresponds to absorption length, $l_{a}$ $=980$ to $98 \mu \mathrm{m}$. The diameter of polystyrene spheres was $0.45 \mu \mathrm{m}$ (Sekisui Chemical Co.) [15]. The volume fraction of polystyrene spheres was changed from $2 \%$ to $10 \%$. These volume fractions correspond to the transport mean-free-path of 100 to $20 \mu \mathrm{m}$, respectively. The sample cell was a $10 \mathrm{~mm} \times 10 \mathrm{~mm}$ quartz cell with a magnetic stirrer to avoid the sedimentation and the thermal effects. The luminescence light from the incident surface of sample cell was collected in a reflection geometry and led to a $25 \mathrm{~cm}$ spectrometer (SM), and detected by a charge coupled device (CCD) camera. Special care was taken to collect all luminescence light from the incident surface of sample cell properly, since, in our experiments, the focusing position of the excitation beam was scanned during the measurement of the luminescence spectra. Figure 2 shows the spectral width of the luminescence light excited with a single beam as a function of excitation pulse energy. Samples were $2 \times 10^{-4} \mathrm{M}, 5 \times 10^{-4} \mathrm{M}$, and $10 \times 10^{-4} \mathrm{M}$ in the dye concentration and $2 \%, 5 \%$, and $10 \%$ in the volume fraction of the polystyrene spheres. With increasing the excitation pulse energy, the spectral width collapses owing to the strong stimulated emission in the sample $[2,5,13,14]$. We define the threshold of the spectral collapse as the excitation pulse energy $W_{t h}$, at which the spectral width becomes half of its initial value. Three arrows in Fig. 2 show the excitation pulse energy at which the gain volume was examined using the two-beam spatial-correlation method. Figure 3 shows the luminescence spectra of the sample of $5 \times 10^{-4} \mathrm{M}$ in the dye concentration and $5 \%$ in the volume fraction of polystyrene spheres in the two-beam spatial-correlation method. The dashed line represents luminescence spectrum observed with the first beam only in the absence of the second beam, while the dotted line is that observed with the second beam. The solid line represents luminescence spectrum observed when both of the first and the second beams were injected simultaneously under a condition: $d=0$. It is seen that with two excitation beams, the spectral width was reduced to be $11 \mathrm{~nm}$. This indicates that

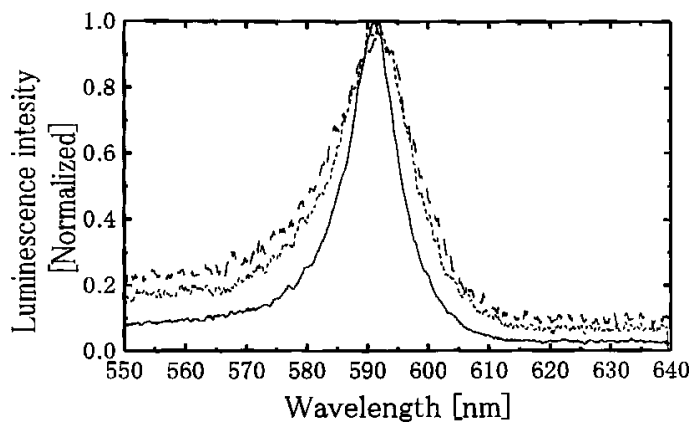

FIG. 3. Luminescence spectra of $5 \times 10^{-4} \mathrm{M}, 5 \%$ sample in the two-beam spatial-correlation method. The dashed line represents luminescence spectrum observed with the first beam, while the dotted line is that observed with the second beam. The solid line represents luminescence spectrum observed when both of the first and the second beams were injected simultaneously.

the two beams work cooperatively to result in the strong stimulated emission in the sample. Figure 4 shows the spectral width $S W(d)$, as a function of the beam separation $d$, between the first and second beams. With increasing the beam separation the spectral width becomes wide. For example, in $5 \times 10^{-4} \mathrm{M}, 5 \%$ sample, when $d \rightarrow \infty$, the spectral width approaches $16 \mathrm{~nm}$, which is the value observed with the single-beam excitation. This indicates that spatially separated two beams as $d \rightarrow \infty$ excite the sample independently [15]. We define the correlation length $L_{c}$ as the length that the spectral width becomes $S W\left(L_{c}\right)=\{S W(0)+S W(\infty)\} / 2$. We performed similar experiments for different samples of dye concentrations and volume fractions of the polystyrene spheres. The results are summarized in Table I, where $N$ is dye concentration, $l_{a}=1 / \sigma_{a} N$ the absorption length, $\sigma_{a}$ the absorption cross section, $l^{*}$ the transport mean-free-path, and $L_{a}=\sqrt{l^{*} l_{a} / 3}$ the diffusive absorption length. In order to deconvolute the effect of the excitation beam size, we also define the reduced correlation length, $L_{c}^{*}=\sqrt{L_{c}^{2}-w^{2}}$, where $w$ is the beam diameter and $w=80 \mu \mathrm{m}$ in our experiments. Figure 5 shows correlation length $L_{c}^{*}$, as a function of $L_{a}$. It is seen that $L_{c}^{*}$ is proportional to $L_{a}$ with a proportional factor of 5.0.

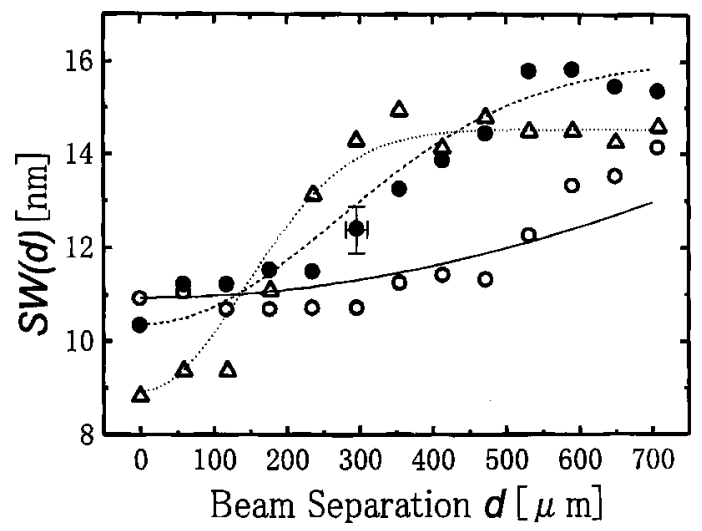

FIG. 4. Luminescence spectral width $S W(d)$ as a function of the beam separation $d$. The concentrations of dye are $(\bigcirc) 2 \times 10^{-4} \mathrm{M}$, (○) $5 \times 10^{-4} \mathrm{M}$, and $(\triangle) 10 \times 10^{-4} \mathrm{M}$ with the volume fractions of polystyrene spheres $2 \%, 5 \%$, and $10 \%$, respectively. The solid, dashed, and dotted lines are corresponding calculated curves. 
TABLE I. Relation between correlation length and some parameters.

\begin{tabular}{lccccccc}
\hline \hline Sample & $\begin{array}{c}N \\
\left(\times 10^{-4} \mathrm{M}\right)\end{array}$ & $\begin{array}{c}l_{a} \\
(\mu \mathrm{m})\end{array}$ & $\begin{array}{c}l^{*} \\
(\mu \mathrm{m})\end{array}$ & $\begin{array}{c}L_{a} \\
(\mu \mathrm{m})\end{array}$ & $\begin{array}{c}L_{c} \\
(\mu \mathrm{m})\end{array}$ & $\begin{array}{c}L_{c}^{*} \\
(\mu \mathrm{m})\end{array}$ & $L_{c}^{*} / L_{a}$ \\
\hline No. 1 & 2 & 490 & 100 & 128 & 684 & 672 & 5.3 \\
No. 2 & 1 & 980 & 20 & 81 & 570 & 555 & 6.9 \\
No. 3 & 5 & 196 & 40 & 51 & 372 & 349 & 6.9 \\
No. 4 & 5 & 196 & 20 & 36 & 290 & 259 & 7.2 \\
No. 5 & 10 & 98 & 20 & 26 & 208 & 163 & 6.3 \\
\hline \hline
\end{tabular}

To explain the experimental results, we performed numerical simulations. The energy states for dyes are the ground $S_{0}$, and excited state $S_{1}$, which consist of many vibrational sublevels. Since the nonradiative decay rates within $S_{0}$ and $S_{1}$ manifolds are very fast, we assume that the population of $S_{0}$ and $S_{1}$ are in their lowest sublevels. Basic equations used are rate equations for the population in dye molecules

$$
\begin{aligned}
\frac{\partial N_{1}(r, t)}{\partial t}= & \sigma_{a}\left(\lambda_{e}\right) N_{0}(r, t) c \Phi_{e}(r, t) \\
& -\int \sigma_{s}\left(\lambda_{l}\right) N_{1}(r, t) c \Phi_{l}\left(\lambda_{l}, r, t\right) d \lambda_{l}-\frac{N_{1}(r, t)}{\tau},
\end{aligned}
$$

and the diffusion equation for the excitation light density $\Phi_{e}(r, t)$,

$$
\frac{\partial \Phi_{e}(r, t)}{\partial t}=\frac{c l^{*}}{3} \nabla^{2} \Phi_{e}(r, t)-\sigma_{a}\left(\lambda_{e}\right) N_{0}(r, t) c \Phi_{e}(r, t),
$$

and luminescence light density $\Phi_{l}\left(\lambda_{l}, r, t\right)$,

$$
\begin{aligned}
\frac{\partial \Phi_{l}\left(\lambda_{l}, r, t\right)}{\partial t}= & \frac{c l^{*}}{3} \nabla^{2} \Phi_{l}\left(\lambda_{l}, r, t\right) \\
& +\sigma_{s}\left(\lambda_{l}\right) N_{1}(r, t) c \Phi_{l}(\lambda, r, t)+\frac{N_{1}(r, t)}{\tau} L\left(\lambda_{l}\right),
\end{aligned}
$$

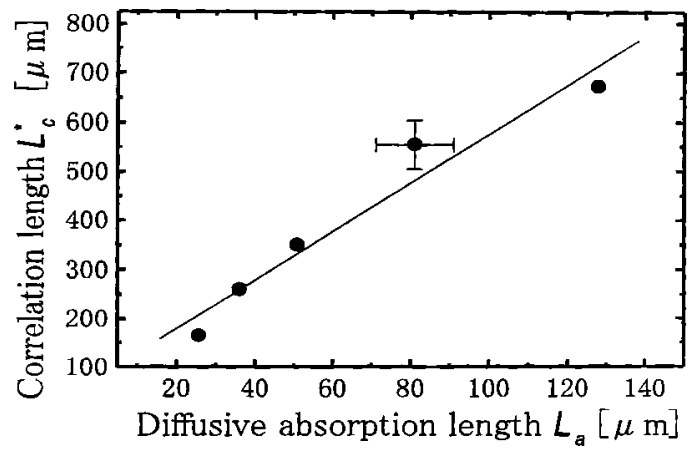

FIG. 5. Correlation length $L_{c}^{*}$, as a function of diffusive absorption length $L_{a}$. The solid line represents fitting line with a proportional factor 5.0.

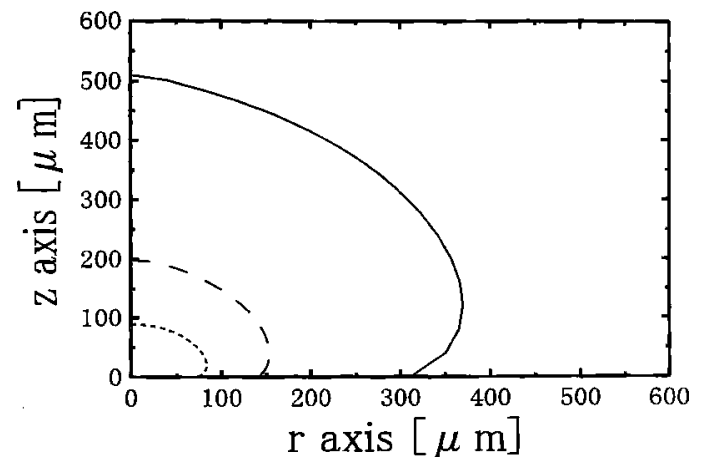

FIG. 6. The contour map for the normalized excited state population of 0.5 under the excitation pulse energy of $W_{t h}$. The concentrations of dye and the volume fractions of polystyrene for solid, dashed, and dotted lines are $2 \times 10^{-4} \mathrm{M}, 2 \% ; 5 \times 10^{-4} \mathrm{M}, 5 \% ; 10$ $\times 10^{-4} \mathrm{M}, 10 \%$, respectively. The $\mathrm{r}$ axis is the distance from the center of the excitation beam on the incident surface. The $\mathrm{z}$ axis is the depth from the incident surface.

where $N_{0}$ and $N_{1}$ are ground-state and excited-state population of dye molecules, respectively, $\lambda_{e}$ and $\lambda_{l}$ are wavelengths of excitation and luminescence light, respectively, $c$ is the light velocity, $\tau$ is the excited state lifetime, and $L\left(\lambda_{l}\right)$ is the spectral shape of spontaneous emission. Parameters used in our calculation were as follows. The absorption cross section for $532 \mathrm{~nm}$ excitation light $\sigma_{a}$, was $1.7 \times 10^{-20} \mathrm{~m}^{2}$. The stimulated emission cross section $\sigma_{s}$, was 1.0 $\times 10^{-20} \mathrm{~m}^{2}$. The excited-state lifetime $\tau$, was $3.6 \mathrm{~ns}$. In Fig. 2 , the solid, dashed, and dotted lines present the numerically calculated spectral width as a function of excitation pulse energy with a single beam excitation. The simulations show good agreement with experimental results for three samples. We developed our simulation model so that we can calculate the luminescence spectral width as a function of the beam separation $d$. The results are shown in Fig. 4 together with the experimental results. The pulse energies indicated by arrows in Fig. 2 were used for the first and the second beams. These results show good agreements with the experimental results. Figure 6 shows the calculated curves for the spatial distribution of the excited-state population of dye molecules in the medium. The lines represent the contour map. We define $L_{t h}$ as the full width that the normalized excited-state population becomes 0.5 in the calculation shown in Fig. 6. It is found that the $L_{c}^{*}$ obtained from the two-beam spatialcorrelation experiment shown in Fig. 4 is in agreement with $L_{t h}$ in Fig. 6 for all samples. We can understand that the correlation length $L_{c}^{*}$ reflects $L_{t h}$.

Let's consider the relation between $L_{c}^{*}$ and $L_{a}$ shown in Fig. 5. The typical path length for the luminescence light to travel in the gain region under the excitation pulse energy of $W_{t h}$ is

$$
\xi_{t h}=\frac{\left(L_{t h}\right)^{2}}{l^{*}}
$$

The gain-length product that the luminescence light obtains during the diffusing process may be $\sigma_{s} N \xi_{t h}$. At the thresh- 
old, this product should be equal to $G_{t h}$, where $G_{t h}$ is the threshold gain to overcome the loss in the medium. Therefore,

$$
L_{t h}=\sqrt{3 G_{t h}} \sqrt{\frac{\sigma_{a}}{\sigma_{s}}} L_{a} .
$$

From Eq. (5), it is seen that the ratio of $L_{t h}$ to $L_{a}$ is proportional to the square root of the ratio of the absorption cross section $\sigma_{a}$, to the stimulated emission cross section $\sigma_{s}$. Since dye molecules used in our experiments was a single kind of dye, kiton red, the value of $\sqrt{\sigma_{a} / \sigma_{s}}$ is constant and $\sqrt{\sigma_{a} / \sigma_{s}}=1.3[16]$. We consider that $L_{c}^{*}$ obtained in the twobeam correlation measurement reflects $L_{t h}$ as discussed before. Therefore, the relation between $L_{c}^{*}$ and $L_{a}$ shown in Fig. 5 is well explained in this model. From Fig. $5, L_{c}^{*}$ at the threshold is five times longer than $L_{a}$. This means that absorption saturation and the resultant increment in the gain volume are essential in the spectral collapse of the random laser. The proportional factor of 5.0 in Fig. 5 would reflect $G_{t h}$, which is subject to the loss of the luminescence light in the medium due to the reabsorption, scattering, and geometrical configurations. From the value $\sqrt{\sigma_{a} / \sigma_{s}}=1.3$ for kiton red and the proportional factor of 5.0, $G_{t h}$ is estimated to be 1.2 , that means $G_{t h} \sim 1$, which is a reasonable value for the gain threshold.

In summary, we measured the size of the gain volume in an amplifying and scattering medium using the two-beam spatial-correlation method. It was found that $L_{t h}$ is proportional to $L_{a}$. Numerical simulation based on the coupled rate and diffusion equations recaptures the experimental results. These results are well explained when we consider the diffusive propagation of light in the medium in the presence of absorption saturation.

This work was supported by Casio Since Foundation and Hamamatsu Science and Technology Foundation. The authors are grateful to Dr. K. Totsuka for his help in writing the program.
[1] R.V. Ambartsumyan, P.G. Krykov, and V.S. Letokhov, Zh. Éksp. Teor. Fiz. 51, 1669 (1966) [Sov. Phys. JETP 24, 1129 (1967)].

[2] M.N. Lawandy, R.M. Balachandran, A.S.L. Gomes, and E. Sauvain, Nature (London) 368, 436 (1994).

[3] W.L. Sha, C.-H. Liu, and R.R. Alfano, Opt. Lett. 19, 1922 (1994).

[4] W. Zhang, N. Cue, and K.M. Yoo, Opt. Lett. 20, 961 (1995).

[5] R.M. Balachandran, N.M. Lawandy, and J.A. Moon, Opt. Lett. 22, 319 (1997).

[6] G. Backering, S.J. Zilker, and D. Haarer, Opt. Lett. 22, 1427 (1997)

[7] D.S. Wiersma, M.P. van Albada, B.A. van Tiggelen, and A. Lagendijk, Phys. Rev. Lett. 74, 4193 (1995).

[8] D.S. Wiersma and A. Lagendijk, Phys. Rev. E 54, 4256 (1996).
[9] H. Cao, Y.G. Zhao, S.T. Ho, E.W. Seelig, Q.H. Wang, and R.P.H. Chang, Phys. Rev. Lett. 82, 2278 (1999).

[10] A.Z. Genack and J.M. Drake, Nature (London) 368, 400 (1994).

[11] K. Totsuka, M.A.I. Talukder, and M. Tomita, J. Phys. Soc. Jpn. 68, 307 (1998).

[12] K. Totsuka, M.A.I. Talukder, M. Matsumoto, and M. Tomita, Phys. Rev. B 59, 50 (1999).

[13] K. Totsuka, G. van Soest, T. Ito, A. Lagendijk, and M. Tomita, J. Appl. Phys. 87, 7623 (2000).

[14] G. van Soest, M. Tomita, and A. Lagendijk, Opt. Lett. 24, 306 (1999).

[15] M. Tomita, K. Totsuka, and H. Ikari, J. Opt. Soc. Am. B 16, 1951 (1999).

[16] F.P. Schäfer, Dye Lasers (Springer-Verlag, Berlin, 1977). 